\title{
A Novel Prioritised Concealment and Flexible Macroblock Ordering Scheme for Video Transmission
}

\author{
T. P. Fowdur \\ Department of Electrical and \\ Electronic Engineering \\ University of Mauritius
}

\author{
D. Indoonundon \\ Department of Electrical and \\ Electronic Engineering \\ University of Mauritius
}

\author{
K. M. S Soyjaudah \\ Information \& Communication \\ Technologies Authority of \\ Mauritius
}

\begin{abstract}
Video compression standards such as H.264/AVC and H.265/HEVC are widely used in applications such as video conferencing, streaming and television broadcasting. These compression standards have been ubiquitously adopted due to their high compression performance as compared to previous standards. However, compressed video may suffer from severe degradation during transmission over unreliable channels due to packet losses which can result in low Quality of Service (QoS). In this paper, a new Space Time Flexible Macroblock Ordering (ST-FMO) scheme is proposed which considers both spatial and temporal re-mapping of Macroblocks(MBs) within a Group of Pictures (GOP). Moreover, a new prioritisation method to determine the concealment order of lost blocks is proposed. The new method uses the autocorrelation function as a measure to determine which blocks should be concealed first. The combined application of the novel ST-FMO and prioritisation scheme provides average gains of over of $2.34 \mathrm{~dB}$ over a conventional scheme and $1.11 \mathrm{~dB}$ over an existing FMO and prioritisation scheme.
\end{abstract}

\section{General Terms}

Video Transmission

\section{Keywords}

Video Compression, ST-FMO, Prioritisation, Concealment.

\section{INTRODUCTION}

H.264 and H.265 are popular video coding standards in use today and developed by the ITU-T Video Coding Experts Group and the ISO/IEC Moving Picture Experts Group [1-4]. Due to its high compression efficiency, H.264 has recently been investigated for live streaming over Enhanced Multimedia Broadcast Multicast Service (e-MBMS)[5]. Recently, in [6] the authors presented a novel fast intra prediction mode decision method for H.264/AVC which uses the prediction directions and the Sum of Absolute Transformed Differences (SATD) of the recovered neighbouring pixels and block content to eliminate some of the least likely prediction modes from the Rate Distortion Optimization (RDO) computation. The results showed that this new method decreased the coding time with little video quality degradation and bit rate increment [6]. In addition, the use of H.264/AVC codec with 4G-LTE technology has proved to be very efficient by reducing the bandwidth for video transmission and at the same time rendering good quality video [7]. H.264 uses advanced prediction algorithms whereby a pixel value can be estimated by using either surrounding pixels (spatial prediction) or pixels from the previous frame or future frames (temporal prediction) in order to achieve higher compression $[4,8]$. However, transmission of video over wired and wireless communication channels is a very challenging task since compressed video is highly sensitive to transmission errors. Due to the prediction algorithms used in H.264, the errors are propagated into subsequent frames within a Group of Pictures, which consequently result in severe degradation in the quality of the video [8]. In addition, a major issue associated with applications such as video streaming over the internet is burst errors resulting into packet losses. Burst errors which occur frequently over congested networks can significantly degrade quality of a video and consequently result in poor QoS and Quality of Experience (QoE) [9-10]. Therefore, it is vital for a video compression standard to cater for packet losses during video transmission $[3,11]$. A popular method of dealing with this problem is to use Flexible Macroblock Ordering (FMO) which is a very efficient error resilient tool in H.264. The idea behind FMO is to reorder Macroblocks (MBs) in such a way that it efficiently improves the concealment of lost MBs [12]. Moreover, several recent studies have shown that prioritising the concealment order can significantly enhance the quality of a video. An overview of FMO as well as prioritisation techniques developed for H.264 video transmission is given next.

Many FMO techniques have been extensively studied and developed in recent years. For example in [13], FMO was used for the rearrangement of MBs into slice groups based on the content of the message bits. Results have shown that the proposed technique surpasses previous works in terms of message payload which leads to reduced distortion and compression overhead [13]. In [12], an Explicit ChessboardWipe (ECW) FMO scheme was proposed which defines a new ordering mechanism of the MBs. This new technique proved to outperform all the FMO types by maximising the number of correctly received Macroblock surrounding a corrupted Macroblock by surpassing the existing Chessboard FMO type by an average gain of $1.52 \mathrm{~dB}$ [12]. In [14], a new technique was proposed which considers the importance of each $\mathrm{MB}$ in order to generate a FMO explicit map. Furthermore, the intra refresh rate was used for diminishing dependency between frames to prevent error propagation. The proposed method outperformed previous methods in terms of PSNR [14].

Prioritisation of the concealment order is another efficient technique to minimise the effect of channel errors. For example in [15] an algorithm was used to determine the importance of each Macroblock so as to determine the order of concealment of the MBs. The importance of the lost macroblock was determined by calculating the impact the loss MBs has on the subsequent frames. This technique is useful only when temporal concealment is used. In addition, the authors in [16] proposed a novel exemplar based spatial error concealment scheme which uses adaptive ordering block match. The order of the concealment is determined by the 
intensity, reliability and similarity of the surrounding blocks [16]. The results showed that the proposed technique achieves performance gains of 1.6 to $1.8 \mathrm{~dB}$ on quality as compared to an existing algorithm which was implemented in JM14.0 [16]. However, the method for determining the concealment order was applicable to only spatial concealment.

In this paper, ST- FMO and Prioritisation schemes for H.264 transmission are proposed. The novel Space Time FMO (ST-FMO) scheme reorders the MBs in such a way that it increases the number of spatially and temporally available neighbours surrounding the lost MBs thereby significantly enhancing both spatial and temporal concealment. In the proposed prioritisation scheme, the order in which the MBs will be concealed is determined by an autocorrelation algorithm. This algorithm exploits the correlation between both neighbouring pixels and previous frames in order to determine the order of concealment as compared to previous prioritisation techniques where either spatial or temporal concealment was used. Simulations results have shown that the proposed scheme provides an average gain of $2.34 \mathrm{~dB}$ compared a conventional scheme and an average gain of 1.11 $\mathrm{dB}$ over an existing FMO and prioritisation schemes. It is to be noted that existing FMO techniques reorder the MBs within each frame which is efficient when only spatial concealment is used. However, the proposed FMO technique increases the available spatial and temporal neighbours which improves both spatial and temporal concealment and therefore provide better video quality. Finally, previous works on prioritisation have focused on either spatial or temporal levels, whereas the proposed prioritisation algorithm works at both the spatial and temporal levels.

This remainder of this paper is organized as follows. Section 2 provides background information on existing FMO and prioritization

schemes.

Section 3 presents the proposed framework and its design. Section 4 demonstrates the experimental results and finally in Section 5 draws some conclusions and scope for future works.

\section{BACKGROUND}

This section describes the basic concepts behind FMO and prioritization schemes.

\subsection{Flexible Macroblock Ordering}

Flexible Macroblock Ordering (FMO) has proved to be one of the most remarkable error-resilient tool within the H.264/AVC standard. In previous video compression standards, MBs were attributed to slices in a raster scan manner [11-18]. With the FMO approach, MBs are no longer grouped into slices but grouped according to a technique referred as slice groups [1118]. Therefore, each macroblock is assigned to a slice group by using a Macroblock Allocation map (MBAmap), thus, ensuring that neighbouring MBs are not found in the same slice group[17-18]. This approach aims to enhance the concealment of a lost $\mathrm{MB}$ from its correctly received neighbouring MBs by reducing the impact of consecutive MB loss which is usually the result of burst channel errors [12]. The H.264 standard describes seven predefined types of FMO referred as Type 0 to Type 6 [18]. One of the most popular FMO techniques is known as chessboard FMO. In [12], the authors proposed a combination type 0 and type 5 FMO known as Explicit Chessboard Wipe (ECW) FMO which outperforms all the other FMO types.

Figure 1 illustrates a decoded frame from the foreman sequence which has been transmitted over a lossy channel at a packet loss rate of 0.2 using the Gilbert Elliot channel model[19-20]. Figure 1(a) is encoded without FMO while
Figure 1(b) and Figure 1(c) have been encoded using chessboard FMO and ECW FMO [12]. Reconstruction using raster scan as shown in Figure 1(a) is quite challenging compared to Figure 1(b) and Figure 1(c) where chessboard and ECW FMO [12] have been used respectively.

From the analysis, it can be concluded that Figure 1(c) provides the highest PSNR of $11.1879 \mathrm{~dB}$. This is because with ECW FMO [12] the errors are more de-localised throughout the frame which consequently increases the number of neighbouring MBs. In addition, when error concealment is employed along with FMO, further gains are obtained. The effect of concealment with FMO can be seen in Figure 1(d), Figure 1(e) and Figure 1(f) which in fact represent the concealed version of frames 1,2 and 3 respectively. The Lagrange Interpolation [21] technique has been used as temporal concealment of the frame in this example. It can be observed that Figure 1(f) provides a maximum gain of $23.1920 \mathrm{~dB}$ after concealing Frame 3 .

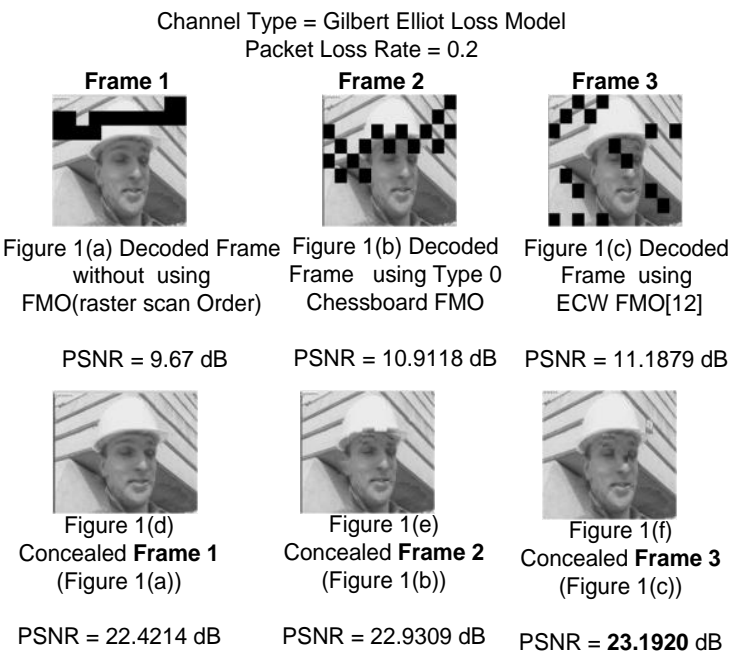

Fig 1: Decoded / concealed frames using FMO schemes

\subsection{Prioritised Concealment}

An effective approach to combat the effect of packet loss over congested networks is Error Concealment. Many error concealment techniques have been extensively studied over the years. The main objective of error concealment is to repair lost or corrupted MBs after its transmission over the communication channel. In addition, prioritisation of error concealment determines the order of the concealment process of the MBs. The Impact Factor algorithm developed in [15] is an efficient technique used for determining the influence of each lost $\mathrm{MB}$ on the video sequence. This algorithm states that the impact of a $\mathrm{MB}$ in a frame is determined by the concealment algorithm which is used to repair the corrupted $\mathrm{MB}[15]$. With the use of the equations described in [15], the impact of each $\mathrm{MB}$ is calculated and the order of their concealment is determined, therefore ensuring an efficient concealment of corrupted MBs.

\section{PROPOSED SYSTEM}

This paper proposes a novel FMO technique which increases the number of spatially and temporally available neighbouring MBs. In addition, this paper also presents a new prioritisation scheme using auto-correlation of neighbouring pixels which determines the order in which the MBs will be concealed. Frequency selective extrapolation (FSE) [22] and Lagrange Interpolation (LI) [21] have been used for error concealment. Figure 2 shows the encoder part of the proposed scheme. The 
input to the system consists of a video sequence containing $N$ frames. First, the $N$ frames are converted into $M$ GOPs each of length $L$ such that [8]:

$$
N=M \times L
$$

Each GOP is processed independently by the H.264 Encoder. The new FMO scheme referred as Space-Time FMO (STFMO) is applied to encoded bitstream before transmission over the Gilbert Elliot Channel [19-20].

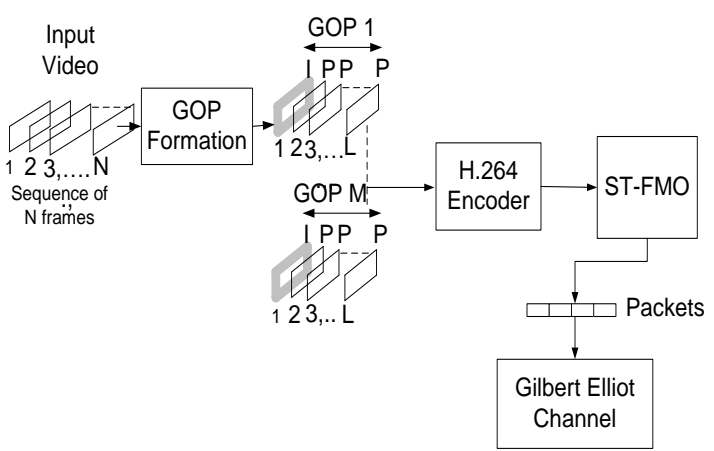

Fig 2: Proposed Encoder with ST-FMO

Figure 3 represents the decoder for the proposed scheme. Inverse ST- FMO is first applied to the bit stream so that the packets (representing the MBs) are re-organised in order to restore the initial positions of the packets. A new prioritisation process is then used to calculate the relative importance of each corrupted MBs by using the correlation between the available neighbouring pixels. This will be described afterwards. Based, on their importance, the order in which the MBs will be concealed is determined, hence, allowing a more efficient concealment. In the final stage, a concealment algorithm is invoked in order to conceal the lost MBs. The concealment algorithm can either be spatial or temporal and is selected adaptively using a concealment selection algorithm.

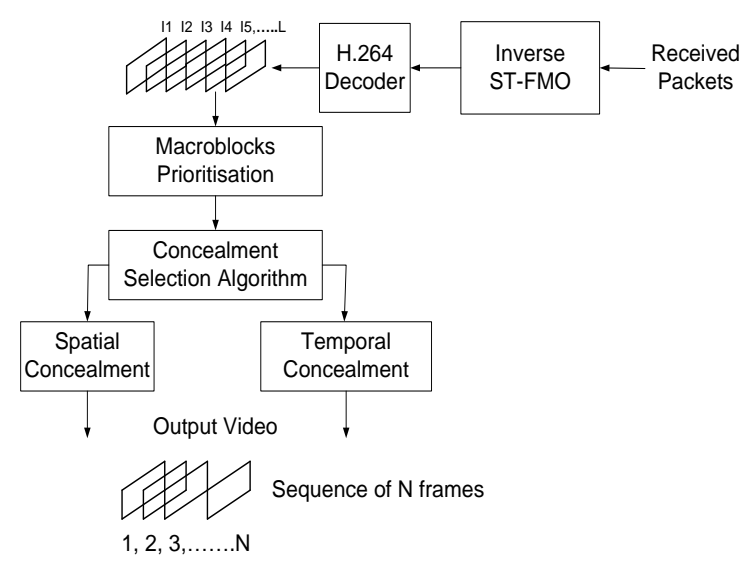

Fig 3: Proposed Decoder with ST FMO and Prioritised Concealment

\subsection{Proposed ST-FMO technique}

Figure 4 illustrates the ST-FMO scheme.
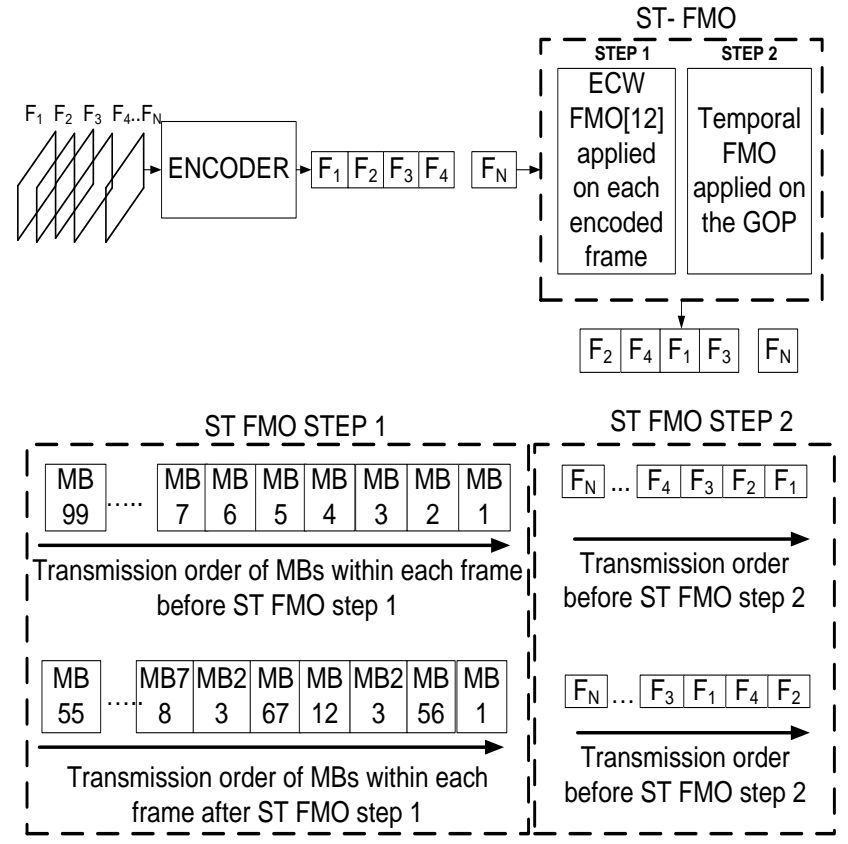

Fig 4: Proposed ST- FMO

The main objective of FMO is to increase the number of spatial neighbours within a frame in order to improve the concealment of lost MBs. However, given the fact that MBs located in the previous or next frame are more significant as compared to neighbouring spatial MBs, this paper proposes ST- FMO which considers both spatial and temporal MBs.

This technique operates in two steps. It first uses the ECWFMO scheme of [12] to achieve spatial FMO within an encoded frame. After this stage the MBs within the frame are re-ordered as in [12]. Secondly, it shuffles the frames in a GOP such that no consecutive frame are transmitted together as illustrated in Figure 4. After this stage, no two frames are sent consecutively. For example, Frame 4 is sent after Frame 2 and Frame 1 after Frame 3.

Consider a loss scenario whereby MBs in Frame 1, Frame 4 and Frame 6 experience burst errors. The transmission order is shown in Figure 5. It can be observed that Frame 1, Frame 4 and Frame 6 experience burst errors. Since Frame 1 is an IFrame spatial concealment can be used to conceal the lost MBs. With the re-arrangement of the MBs within a frame using ECW-FMO of [12], the number of available MBs is increased which results in a more effective spatial concealment. With ST-FMO, the order of transmission of the frames within a GOP changes. For example, in Figure 5(b) it can be seen that Frame 5 and Frame 6 are not transmitted consecutively. Since Frame 5 is unaffected by noise it can consequently be used as a reference frame for temporal concealment of Frame 6. In addition, Frame 4 can be concealed by using either Frame 3 (previous frame) or frame 5 (next frame) as reference frame. With spatial FMO alone, this advantage is not obtained. In addition, the proposed STFMO scheme does not have an effect on the encoder performance as ST-FMO is applied to the MBs only after they have been coded into bits. 


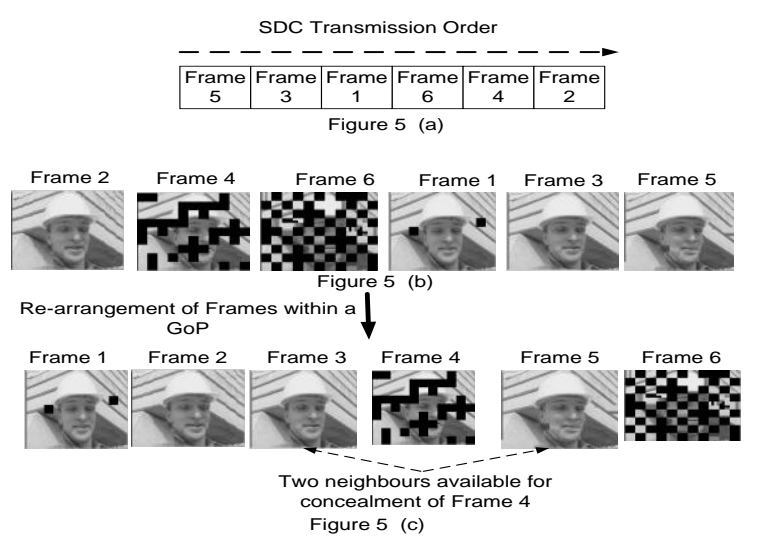

Fig 5: Illustration of a typical loss scenario of a GoP using ST-FMO with Packet Loss Rate of 0.2.

\subsection{Prioritisation using autocorrelation}

The final stage in the proposed framework is Error Concealment. However, prior to concealment this paper proposes a new prioritisation algorithm based on autocorrelation. Using the autocorrelation function, the similarity within a frame i.e. the intraframe autocorrelation is calculated by determining how the pixel similarity varies as a function of distance between the pixels.

Consider Figure 6 in which intraframe autocorrelation is used. $\mathrm{CL}$ represents the lost macroblock which is surrounded by 8 MBs $\mathrm{C}_{\mathrm{A}}, \mathrm{C}_{\mathrm{B}}, \mathrm{C}_{\mathrm{C}}, \mathrm{C}_{\mathrm{D}}, \mathrm{C}_{\mathrm{E}}, \mathrm{C}_{\mathrm{F}}, \mathrm{C}_{\mathrm{G}}$ and $\mathrm{C}_{\mathrm{H}}$. Assume, all the surrounding $8 \mathrm{MBs}$ are correctly received. The correlation, $\mathrm{r}$, is calculated between each two MBs in order to determine the level of similarity between them. For example, $r_{1}$ represents the correlation between $M B s C_{A}$ and $C_{B}, r_{2}$ between $C_{B}$ and $\mathrm{C}_{\mathrm{C}}$ and so on. Therefore, the higher the correlation value between the two MBs, the higher the similarity between them.

The intraframe Autocorrelation algorithm, $r$, is given by equation (2) [23]:

$$
r=\frac{\sum_{m} \sum_{n}\left(P_{p}(u, v)-\mu_{P}\right)\left(Q_{p}(u, v)-\mu_{Q}\right)}{\sqrt{\sum_{m} \sum_{n}\left(P_{p}(u, v)-\mu_{P}\right)^{2} \sum_{m} \sum_{n}\left(Q_{p}(u, v)-\mu_{Q}\right)^{2}}}
$$

Where,

$r$ is correlation coefficient.

$P_{p}(u, v)$ and $Q_{p}(u, v)$ represent two block of pixels.

$u$ and $v$ are pixel positions.

$\mu_{P}$ and $\mu_{Q}$ represent the mean values of $P_{p}(u, v)$ and $Q_{p}(u, v)$ respectively.

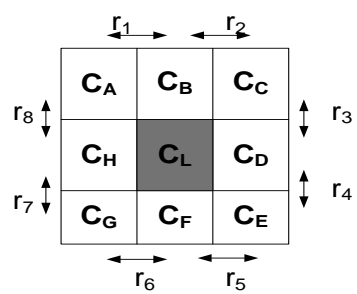

Fig 6: Intraframe Autocorrelation

The average Intraframe autocorrelation, $\bar{C}$ of all the $N_{t}$ MBs surrounding the lost MB can be calculated as follows:

$$
\bar{C}=\frac{1}{N_{t}} \sum_{i=1}^{N_{t}} r_{i}
$$

Where,

$N_{t}$ is the total number of MBs surrounding the lost MBs.

In case, one of the surrounding MBs is also corrupted, the block is replaced by pixel value zero, while computing the value $\mathrm{r}$. The values of $\bar{C}$ are computed for all the lost MBs in an I-frame. Then the one having the highest value of $\bar{C}$ is concealed first. The value of $\bar{C}$ is zero for completely different blocks. Spatial concealment is performed by using the surrounding MBs. Therefore, for an efficient spatial concealment of a lost MB, its neighbouring MBs should be correctly received which is determined by a high correlation value. If a lost MB contains corrupted neighbours as well, the autocorrelation value decreases. This technique ensures an effective way of prioritising the concealment of lost MBs. An illustration of the application of this technique is given in Figure 7.

Figure 7 represents a received frame from a typical foreman sequence which has been transmitted over a Gilbert Elliot Channel at a packet loss rate of 0.4. Consider the three corrupted $\mathrm{MBs} \mathrm{C}_{1}, \mathrm{C}_{2}$ and $\mathrm{C}_{3} . \mathrm{C}_{1}$ is surrounded by 7 correctly received MBs. The average Intraframe autocorrelation, $\bar{C}$ for $\mathrm{C}_{1}$ is 0.5097 .

$\mathrm{C}_{2}$ contains 5 correctly received neighbouring MBs. The value of $\bar{C}$ for $\mathrm{C}_{2}$ is 0.2490 . This is because there is much less similarity between the MBs as it can be seen from Figure 7, $\mathrm{C}_{2}$ is surrounded by 3 lost MBs which therefore decreases its value for $\bar{C} . C_{3}$ is surrounded by correctly received MBs. However, the autocorrelation value is least in this case as $C_{3}$ is positioned in the first row in the frame where upper MBs are not available for concealment. Therefore, $\mathrm{C}_{1}$ will be concealed first as it has the highest correlation value followed by $C_{2}$ and $\mathrm{C}_{3}$ respectively.

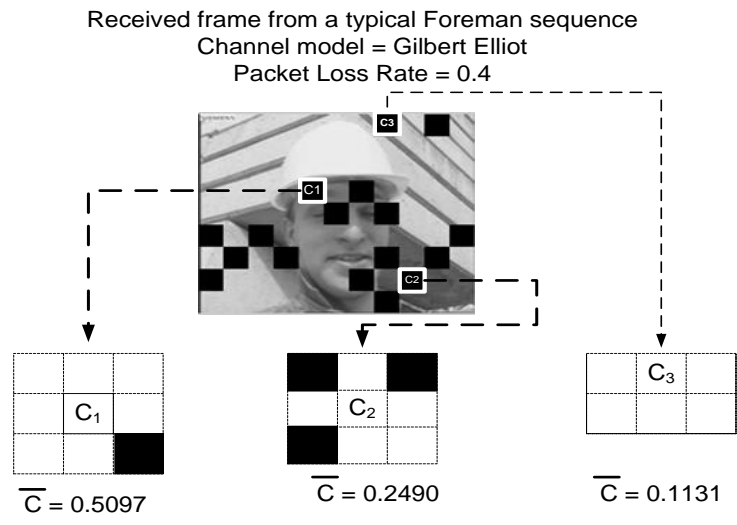

Fig 7: Loss scenarios for calculation of intraframe autocorrelation, $\bar{C}$.

This prioritisation technique can also be extended to temporal correlation whereby interframe correlation, $r_{t}$ is used.

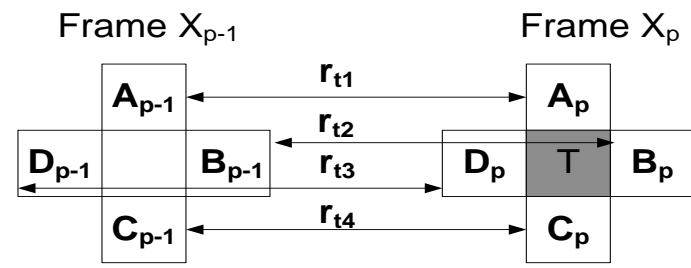

Fig 8: Interframe Autocorrelation

Consider Figure 8 which shows two received frames. $X_{p}$ is the currently received frame and $X_{p-1}$ is the previous frame. $T$ 
represents the lost $\mathrm{MB}$. It is assumed that the concealment algorithm will use $A_{p-1}, B_{p-1}, C_{p-1}$ and $D_{p-1}$ from the reference frame to conceal $\mathrm{T}$.

Since temporal concealment will be used for recovering lost MBs, the similarity between the two frames $X_{p}$ and $X_{p-1}$ is calculated. For example in Figure $8, \mathrm{r}_{\mathrm{t} 1}$ represents the correlation between $\mathrm{MBs} \mathrm{A}_{\mathrm{p}}$ and $\mathrm{A}_{\mathrm{p}-1}$. Along the same line, correlation, $\mathrm{r}_{\mathrm{t} 2}, \mathrm{r}_{\mathrm{t} 3}$ and $\mathrm{r}_{\mathrm{t} 4}$ are calculated using the equation below [23]:

$$
r_{t}=\frac{\sum_{m} \sum_{n}\left(X_{p}(u, v)-\mu_{p}\right)\left(X_{p-1}(u, v)-\mu_{p-1}\right)}{\sqrt{\sum_{m} \sum_{n}\left(X_{p}(u, v)-\mu_{p}\right)^{2} \sum_{m} \sum_{n}\left(X_{p-1}(u, v)-\mu_{p-1}\right)^{2}}}
$$

$r_{t}$ is the correlation coefficient between adjacent frames represented by $X_{p}$ and $X_{p-1}[23]$.

$X_{p}$ represents the current frame.

$X_{p-1}$ represents the previous frame.

Therefore the average Interframe autocorrelation correlation $C_{p}$ is calculated as follows:

$$
C_{P}=\frac{r_{t 1}+r_{t 2}+r_{t 3}+r_{t 4}}{4}
$$

The values $C_{P}$ are computed for all the lost MBs in the Pframes. After that, the one having the highest value of $C_{P}$ is concealed first. For temporal concealment, four neighbouring MBs (top, right, left and bottom) in the reference frame are used. The interframe autocorrelation algorithm checks the similarity between the neighbours of a lost MB in frame $X_{p}$ with those of its reference $M B$ in frame $X_{p-1}$ from the previous frame. Concealment of $X_{p}$ will not be effective if the surrounding MBs are also corrupted. Given the fact that errors are propagated throughout a GOP, it is important to determine the correct order of concealment.

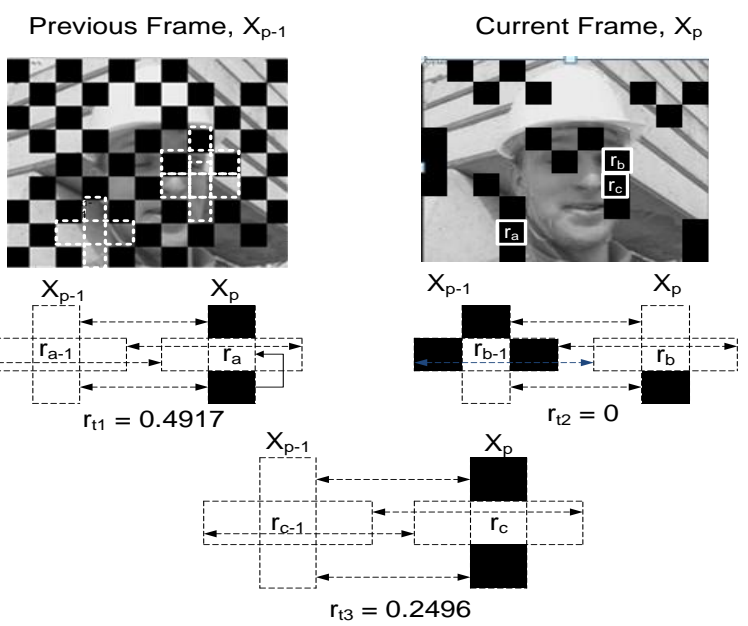

Fig 9: Loss scenarios for calculation of interframe autocorrelation, $\mathbf{C}_{\mathrm{p}}$.

Consider three lost MBs $r_{a}, r_{b}$ and $r_{c}$ in a P-frame $X_{p}$ as shown in Figure 9. $r_{a}$ and $r_{c}$ are both surrounded by two lost MBs and two correctly received MB. The interframe autocorrelation value, $r_{t 1}$ of MB $r_{a}$, is 0.4917 as compared to $r_{c}$ whose interframe autocorrelation value, $r_{t 3}$ is 0.2496 . This is because there is more similarity between the neighbours of $r_{a}$ and $r_{a-1}$. $r_{b}$ is surrounded by three lost MBs and its reference $M B r_{b-1}$ is surrounded by one lost MB. As the neighbouring
MBs of $r_{b}$ are completely different from its reference MB $r_{b-1}$, the interframe autocorrelation value, $\mathrm{r}_{\mathrm{t} 2}$ is zero. Therefore, ra will be concealed first followed by $r_{c}$ and $r_{b}$ respectively.

\subsection{Concealment Selection Process}

The last block of the decoder selects the concealment algorithm to be used which can be spatial or temporal. This is illustrated in Figure 10. First, the algorithm checks whether the frame is an I-frame or a P-frame. If, it is an I-frame the average Intraframe correlation value, $\bar{C}$ is used for determining the order of concealment of the MBs. In the final stage, Frequency Selective Extrapolation (FSE) [22] is used for as spatial concealment of the MBs. Otherwise, if the frame is a $\mathrm{P}$-frame, the Interframe correlation value $\mathrm{C}_{\mathrm{p}}$ is used for determining the concealment order of $\mathrm{MBs}$ and lastly, Lagrange Interpolaton (LI) [21] is used as temporal concealment.

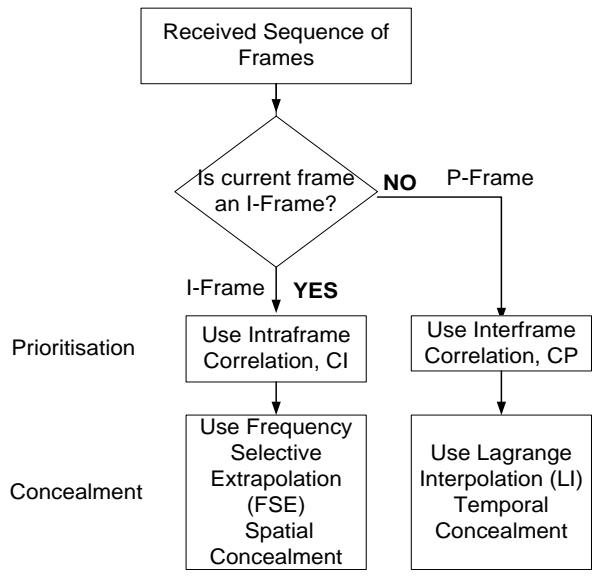

Fig 10: Flowchart illustrating the prioritisation and concealment selection

It is also possible to use other concealment schemes as well as other criteria to select between the use of spatial and temporal concealment.

\section{SIMULATIONS AND RESULTS}

In line with the goals of this work, nine different schemes have been compared. The schemes have been simulated using Matlab with the Foreman and Akiyo video sequences which consists of 300 frames. A Gilbert Elliot Channel Model has been used for all the simulations [19-20]. Each frame is of size $144 \times 176$ pixels and a rate of 25 frames per second has been used. A brief description of each of the schemes is given in Table 1 below:

Table 1. Schemes tested.

\begin{tabular}{|c|c|c|}
\hline Scheme & Type of FMO & Type of Prioritization \\
\hline 1 & ST-FMO & Autocorrelation \\
\hline 2 & ECW-FMO [12] & Autocorrelation \\
\hline 3 & No FMO & Autocorrelation \\
\hline 4 & ST-FMO & Impact Factor [15] \\
\hline 5 & ECW-FMO [12] & Impact Factor [15] \\
\hline 6 & No FMO & Impact Factor [15] \\
\hline 7 & ST-FMO & No Prioritisation \\
\hline 8 & ECW-FMO [12] & No Prioritisation \\
\hline 9 & No FMO & No Prioritisation \\
\hline
\end{tabular}

Scheme 1 represents the proposed framework which uses STFMO and prioritisation based on autocorrelation for concealment. Scheme 2 uses ECW- FMO [12] and prioritisation based on autocorrelation. Schemes 3 and 6 both 
do not employ any FMO technique but use prioritisation based on autocorrelation and Impact Factor [15] respectively for prioritisation. Scheme 4 employs ST-FMO and Impact Factor for prioritisation and Scheme 5 uses ECW-FMO [12] and Impact Factor [15] for prioritisation. Schemes 7 and 8 both do not use any prioritisation method but use ST-FMO and ECW-FMO respectively. Finally, Scheme 9 represents a conventional H.264 transmission scheme without FMO and prioritisation. In all the simulations, FSE [22] is used as spatial concealment and LI [21] is used as temporal concealment.

\subsection{Results with the Foreman sequence using GOP lengths of 6 and 15 .}

Table 2 represents the results obtained using the 9 schemes at packet loss rates of 0.2 and 0.4 with a Foreman video sequence. Moreover, two GOP lengths of 6 and 15 have been used.

Table 2. Results obtained using the Foreman sequence with GOP lengths of 6 and 15 at Packet Loss Rates (PLR) of 0.2 and 0.4 .

\begin{tabular}{|l|l|l|l|l|}
\hline \multirow{2}{*}{ Scheme } & \multicolumn{2}{|c|}{ GOP 6 } & \multicolumn{2}{c|}{ GOP 15 } \\
\cline { 2 - 5 } & PLR = 0.2 & PLR =0.4 & PLR=0.2 & PLR=0.4 \\
\hline Scheme 1 & 17.9967 & 13.3248 & 16.1016 & 12.1475 \\
\hline Scheme 2 & 17.9396 & 13.2064 & 16.0572 & 12.1113 \\
\hline Scheme 3 & 17.8658 & 13.1864 & 15.9753 & 12.1096 \\
\hline Scheme 4 & 17.4495 & 12.2823 & 15.4103 & 11.7549 \\
\hline Scheme 5 & 17.3400 & 12.1530 & 15.3705 & 11.6412 \\
\hline Scheme 6 & 17.2993 & 12.1098 & 15.3453 & 11.5602 \\
\hline Scheme 7 & 16.7456 & 11.9479 & 14.8142 & 11.3364 \\
\hline Scheme 8 & 16.6790 & 11.6793 & 14.7348 & 11.3038 \\
\hline Scheme 9 & 16.5278 & 11.6021 & 14.6225 & 11.2620 \\
\hline
\end{tabular}

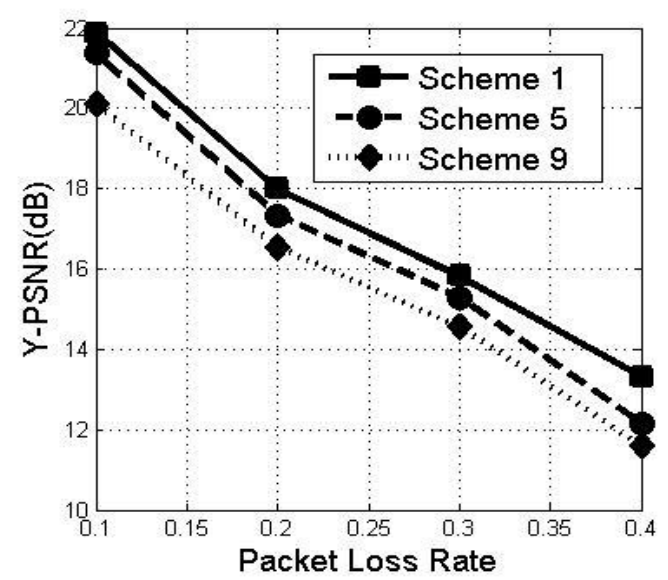

Fig 11: Graph of Y-PSNR against Packet Loss Rate using the Foreman sequence with GOP length $=6$

Figure 11 shows a Graph of Y-PSNR against Packet Loss Rate for the Foreman sequence with GOP length $=6$. It is observed that scheme 1 which uses ST-FMO and autocorrelation as prioritisation provides an average gain of $0.8 \mathrm{~dB}$ over scheme 5 which uses ECW FMO [12] and Impact Factor[15]. In addition, scheme 1 provides an average gain of $1.55 \mathrm{~dB}$ in the range of $0.1 \leq$ Packet Loss Rate $\leq 0.4$ over scheme 9 which does not use FMO and prioritisation. This gain is obtained because ST FMO increases the number of available neighbouring and adjacent MBs which enhances the performance of both spatial and temporal concealments. Furthermore, the prioritisation algorithm provides a more effective way for determining the order of concealment as it considers both autocorrelation between neighbouring and adjacent pixels as well. Scheme 1 achieves a maximum YPSNR value of $21.86 \mathrm{~dB}$ at 0.1 Packet Loss Rate.

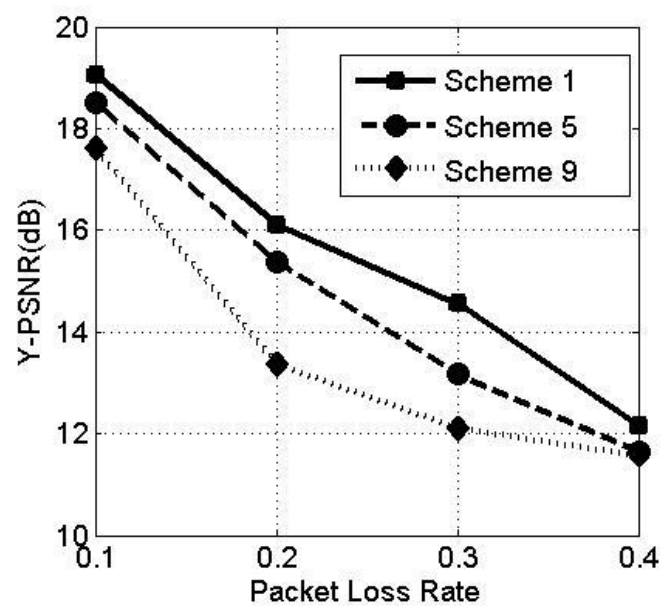

Fig 12: Graph of Y-PSNR against Packet Loss Rate using the Foreman sequence with GOP length $=15$

Figure 12 represents a graph of Y-PSNR against Packet Loss Rate using a GOP length of 15 . It is observed that scheme 1 provides an average gain of $0.79 \mathrm{~dB}$ over scheme 5 in the range of $0.1 \leq$ Packet Loss Rate $\leq 0.4$. Over the same range, Scheme 1 also outperforms Scheme 9 by $0.90 \mathrm{~dB}$. It can be observed that concealment is less effective when the error has been propagated over a larger sequence of frames, i.e. for longer GOP lengths.

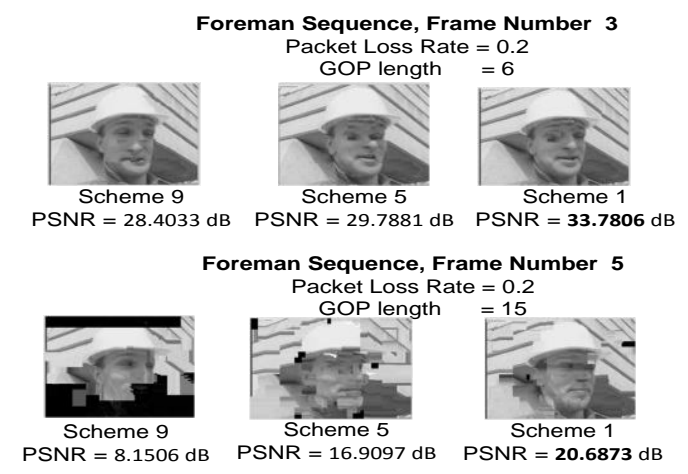

Fig 13: Comparison in Video frame quality between Scheme 1, Scheme 5 and Scheme 9 with Foreman Sequence.

Figure 13 shows frames which have been reconstructed using Scheme 1, Scheme 5 and Scheme 9 with GOP length 6 and 15. Using a GOP length of 6 with frame number 3, it can be seen from the leftmost picture which uses Scheme 9 has a PSNR of $28.4033 \mathrm{~dB}$. The middle picture which uses Scheme 5 provides an additional gain of $1.38 \mathrm{~dB}$ as compared to Scheme 9. The rightmost picture which is reconstructed using the proposed method Scheme 1 outperforms Scheme 5 and Scheme 9 both in terms of PSNR and Visual quality. Using a GOP length of 15 with frame number 5 , it can be observed that the leftmost picture has suffered severe degradation due to both burst errors and error propagation from previous frames. Using Scheme 9, the frame has not been reconstructed effectively with a low PSNR value of $8.1506 \mathrm{~dB}$. With Scheme 5, the errors are de-localised which increases the number of available neighbouring MBs for concealment. Error concealment is more effective with a PSNR value of 
$16.9093 \mathrm{~dB}$. In addition, the proposed technique, Scheme 1 surpasses Scheme 5 and Scheme 9 by providing the highest PSNR value of $20.6873 \mathrm{~dB}$ and acceptable visual quality.

\subsection{Results with the Akiyo sequences using GOP lengths of 6 and 15}

Table 3 represents the results obtained using the 9 schemes at packet loss rates of 0.2 and 0.4 with a Akiyo video sequence. Moreover, two GOP lengths of 6 and 15 have been used.

Table 3. Results obtained using Akiyo sequence with GOP lengths of 6 and 15 at Packet Loss Rates (PLR) of 0.2 and 0.4.

\begin{tabular}{|c|c|c|c|c|}
\hline \multirow{2}{*}{ Scheme } & \multicolumn{2}{|c|}{ GOP 6 } & \multicolumn{2}{c|}{ GOP 15 } \\
\cline { 2 - 5 } & PLR = 0.2 & PLR = 0.4 & PLR = 0.2 & PLR = 0.4 \\
\hline Scheme 1 & 21.1278 & 16.0346 & 16.5245 & 13.4523 \\
\hline Scheme 2 & 20.8942 & 15.8780 & 16.5019 & 13.3980 \\
\hline Scheme 3 & 20.8559 & 15.8315 & 16.4725 & 13.2156 \\
\hline Scheme 4 & 19.6122 & 15.2406 & 14.7020 & 10.7658 \\
\hline Scheme 5 & 19.5078 & 14.8247 & 14.6936 & 10.6789 \\
\hline Scheme 6 & 19.4389 & 14.6345 & 14.6913 & 10.6458 \\
\hline Scheme 7 & 18.5260 & 14.4694 & 13.0396 & 8.8649 \\
\hline Scheme 8 & 18.4630 & 14.3627 & 13.0247 & 8.8221 \\
\hline Scheme 9 & 17.8116 & 14.2861 & 13.0192 & 8.8023 \\
\hline
\end{tabular}

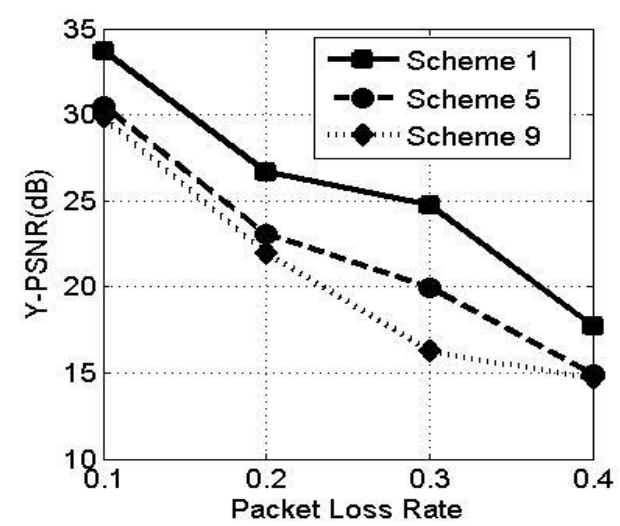

Fig 14: Graph of Y-PSNR against Packet Loss Rate with GOP length $=15$ with GOP length $=6$.

Figure 14 represents a graph of Y-PSNR against Packet Loss Rate for the Akiyo sequence using a GOP length of 6 . It is observed that scheme 1 provides an average gain of $1.11 \mathrm{~dB}$ over scheme 5 in the range of $0.1 \leq$ Packet Loss Rate $\leq 0.4$. Over the same range, Scheme 1 also outperforms Scheme 9 by $2.34 \mathrm{~dB}$.

Figure 15 represents a graph of Y-PSNR against Packet Loss Rate with a GOP length of 15 . It is observed that scheme 1 provides an average gain of $0.90 \mathrm{~dB}$ over scheme 5 in the range of $0.1 \leq$ Packet Loss Rate $\leq 0.4$. Over the same range, Scheme 1 also outperforms Scheme 9 by $2.11 \mathrm{~dB}$.

Figure 16 shows the comparison in visual quality using Scheme 1, Scheme 5 and Scheme 9. It can be seen that Scheme 1 provides better visual quality of the frame and highest PSNR value of $25.8158 \mathrm{~dB}$ using GOP 6 and 20.9810 $\mathrm{dB}$ using GOP 15.

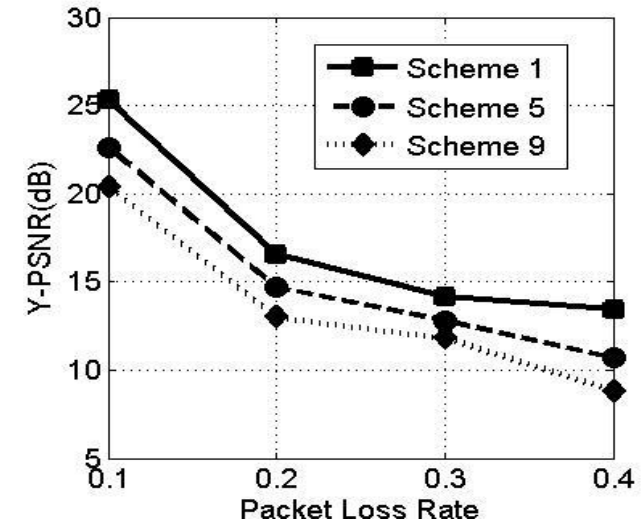

Fig 15: Graph of Y-PSNR against Packet Loss Rate using the Akiyo sequence with GOP length $=15$.

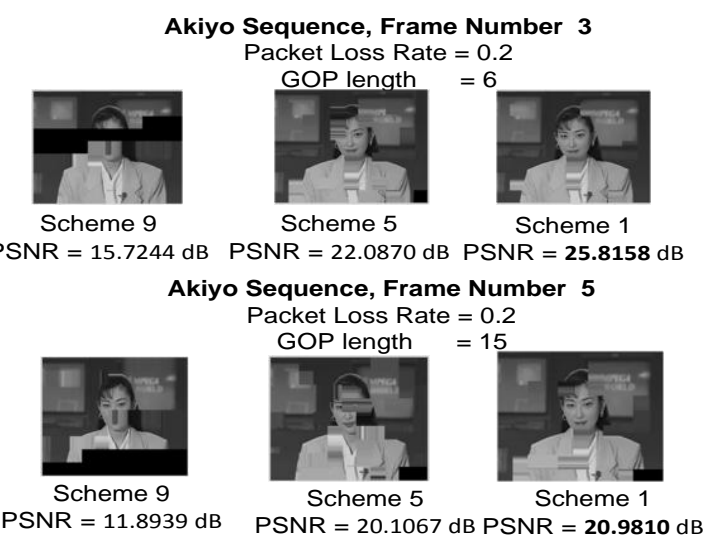

Fig 16: Comparison in Video frame quality using Scheme 1, Scheme 5 and Scheme 9.

\section{CONCLUSION AND FUTURE WORKS}

The aim of this paper is to improve the performance of H.264 video transmission by using a new FMO and prioritisation scheme. A new FMO scheme, ST-FMO, has been proposed which greatly increases the number of available MBs, thereby improving the spatial and temporal concealment of corrupted MBs. The novel combination of ST-FMO has proved to be very useful in combating the influence of packet losses during transmission. Furthermore, a new prioritisation scheme based on autocorrelation is proposed to determine the order of concealment. The prioritisation algorithm is applied to both intraframe and interframe concealment in order to enhance the performance of error the concealment scheme employed. The performance of the proposed scheme was simulated and compared against eight schemes using the Foreman and Akiyo sequences with two different GOP lengths. The proposed scheme provided a significant average gain of $2.34 \mathrm{~dB}$ over a conventional scheme which does not use FMO and prioritisation. The results have shown that the proposed scheme outperforms all the other schemes, hence it is suitable for many real time video applications in order to improve the Quality of Service. The proposed scheme also provides an average gain of $1.11 \mathrm{~dB}$ over an existing FMO and prioritisation scheme. From the results, it can be concluded that the new FMO and prioritisation scheme proved to be an efficient technique in combating burst errors and enhancing error concealment. An interesting future work would be to investigate the optimal GOP length that should be used for a given packet loss rate. Finally the ST-FMO and prioritisation 
scheme proposed can also be applied to H.265 and a performance analysis could be made.

\section{ACKNOWLEDGEMENTS}

The authors are thankful to the University of Mauritius and the Tertiary Education Commission for providing the necessary facilities and financial support for conducting this research.

\section{REFERENCES}

[1] Wiegand, T. 2003. Draft ITU-T Recommendation and Final Draft International Standard of Joint Video Specification (ITU-T Rec. H.264 |ISO/IEC 1449610AVC, ITU-T Rec.H.264 | ISO/IEC 14496-10AVC.

[2] Bross, B. Han, W. J. Ohm, J. R. Sullivan, G. J. and Wiegand, T. 2012. High Efficiency Video Coding (HEVC) Text Specification Draft 9, Joint Collaborative Team on Video Coding (JCT-VC) of ITU-T SG 16 WP 3 and ISO/IEC JTC 1/SC 29/WG 11, document JCTVCK1003, Shanghai, China.

[3] Khan, I. U. Ansari, M. A. Yadav, A. Saeed, S. H. 2015. Performance analysis of H.264 video decoder: Algorithm and applications. In International Conference on Energy Economics and Environment (ICEEE), September 2015, $1-6$.

[4] Wang, H. 2009. 4G wireless video communications, Wiley, Chichester, U.K

[5] Kumar, U. Oyman, O. 2013. QoE Evaluation for Video Streaming over eMBMS. In International Conference on Computing, Networking and Communications.

[6] Mathew, D. and Binish, M.C. 2015. A Fast Intra Prediction for H.264/AVC Based on SATD and Prediction Direction. In International Conference on Emerging Trends in Engineering, Science and Technology, 2015, vol.24, 1016-1023.

[7] Incident Communication Solutions, "Implications of $4 \mathrm{G}$ LTE for First "Iesponders," url:http://incidentcommunications.com/blog/4GLTE\%20-First-Responders

[8] Fowdur, T. Indoonundon, D. Soyjaudah, S. 2014. An enhanced framework for H.264 video transmission with joint prioritisation of retransmission and concealment order. In 9th International Symposium on Communication Systems, Networks \& Digital Signal Processing (CSNDSP), 2014, 634-639.

[9] Al-Jobouri, L. Ali, I. A. Fleury, M. and Ghanbari, M. 2015. Error and Congestion Resilient Video Streaming over Broadband Wireless. In Computers, 2015,vol.4, 113-141.

[10] Rani, U. and Somashekar, K. 2015. An Enhanced Scheme of Video Transmission Using Priority Based Fuzzy Scheduling in Wimax. In International Journal of Electrical and Electronics Research, 2015, vol.3, 126130.

[11] Chen, H. Han, Z. Hu, R. and Ruan, R. 2008. Adaptive FMO selection strategy for error resilient H.264 coding. In International Conference On Audio, Language And Image Processing IEEE, 2008, 868 - 872.

[12] Psannis, K. Ishibashi, Y. 2008. "Efficient Flexible Macroblock Ordering Technique," IEICE Transactions
On Communications, vol.8, 2692-2701. doi:10.1093/ietcom/e91-b.8.2692.

[13] Shanableh, T. 2012. "Data Hiding in MPEG Video Files Using Multivariate Regression and Flexible Macroblock Ordering," IEEE Trans. Inform. Forensic Secur., (April 2012),vol.7, 455-464. doi:10.1109/tifs.2011.2177087.

[14] Thi Huong, T. N. and Huu, T. V. 2015. An improved technique using FMO for error propagation resilience in H.264 video coding. In Information and Computer Science, 2015 2nd National Foundation for Science and Technology, 225-228.

[15] Dhondt, Y. Lambert, P. and Van de Wall, R. 2006. A flexible macroblock scheme for unequal error protection. In Proceedings of the IEEE International Conference on Image Processing, 2006, 829-832.

[16] Zhou, F. Xu, W. and Chen Y. 2013. A Novel Error Concealment Method based on Adaptive Ordering of Block Match. In Proceedings of the 3rd International Conference on Multimedia Technology Volume 278 of the series Lecture Notes in Electrical Engineering, vol. $278,305-314$

[17] Dhondt, Y. Lambert, P. Notebaert, S. and Van de Walle, R. 2005. Flexible macroblock ordering as a content adaptation tool in H.264/AVC. In Proceedings of SPIE, vol. $6015,44-52$.

[18] Dhondt, Y. and Lambert, P. 2004. Flexible Macroblock Ordering an error resilience tool in H.264/AVC, PhD Symposium, Faculty of Engineering, Ghent University.

[19] Gilbert, E. N. 1960. Capacity of a Burst-Noise Channel, Bell System Technical Journal, vol.39, 12531265.doi:10.1002/j.15387305.1960.tb03959.x.

[20] Elliott, E. O. 1963.Estimates of Error Rates for Codes on Burst-Noise Channels, Bell System Technical Journal, vol.42, 1977-1997. doi:10.1002/j.15387305.1963.tb00955.x.

[21] Zheng, J. and Chau, L. P. 2004. A temporal error concealment algorithm for (H.264) using Lagrange interpolation. In Proceedings of the 2004 International Symposium on Circuits and Systems, ISCAS 04, 133136.

[22] Karapetyan G. and Sarukhanyan, H. 2012. On a Modification of the Frequency Selective Extrapolation method. In Information Models and Analyses, vol.1, 139 -145 .

[23] K. Thyagarajan, 2010. Still Image and Video Compresion with Matlab, A John Wiley \& Sons, INC., Publication, 301-305. 